\title{
Altered axon initial segment in hippocampal newborn neurons, associated with recurrence of temporal lobe epilepsy in rats
}

\author{
TIAN-TIAN LIU ${ }^{1}$, LI FENG ${ }^{1}$, HENG-FANG LIU ${ }^{2}$, YI SHU ${ }^{3}$ and BO XIAO ${ }^{1}$ \\ ${ }^{1}$ Department of Neurology, Xiangya Hospital, Central South University, Changsha, Hunan 410008; \\ ${ }^{2}$ Department of Neurology, The Fifth Affiliated Hospital of Zhengzhou University, Zhengzhou, Henan 450052; \\ ${ }^{3}$ Department of Neurology, The Second Xiangya Hospital, Central South University, Changsha, Hunan 410008, P.R. China
}

Received January 6, 2017; Accepted July 6, 2017

DOI: $10.3892 / \mathrm{mmr} .2017 .7017$

\begin{abstract}
Hippocampal neurogenesis in temporal lobe epilepsy (TLE) may result in alteration of the excitability of neurons, which contributes to spontaneous recurrent seizures. Axon initial segment (AIS) structural and functional plasticity is important in the control of neuronal excitability. It remains to be elucidated whether the plasticity of AIS occurs in hippocampal newly-generated neurons that are involved in recurrent seizures following pilocarpine-induced status epilepticus (SE). The present study first established a pilocarpine-induced TLE rat model to assess the features of newborn neurons and AIS plasticity alterations using double immunofluorescence staining of Ankyrin G and doublecortin (DCX). AIS plasticity alterations include length and distance from soma in the hippocampal newly-generated neurons post-SE. The results of the present study demonstrated that pilocarpine-induced epileptic rats exhibited aberrant hippocampal neurogenesis and longer DCX-labeled cell dendrites in the dentate gyrus. Pilocarpine-induced epileptic rats demonstrated shortened lengths of AIS and an increased distance from the soma in hippocampal newborn neurons. Mibefradil, a T/L-type calcium blocker, reversed the alterations in length and position of AIS in hippocampal newborn neurons post-SE, accompanied by decreased long-term seizure activity without increased aberrant neurogenesis. These findings indicate that the plasticity of AIS in hippocampal neurogenesis may have profound consequences in epilepsy, at least in animals.
\end{abstract}

Correspondence to: Dr Li Feng or Dr Bo Xiao, Department of Neurology, Xiangya Hospital, Central South University, 87 Xiangya Road, Changsha, Hunan 410008, P.R. China

E-mail: fengli1982@yahoo.com.cn

E-mail: xiaobo_xy@126.com

Abbreviations: TLE, temporal lobe epilepsy; AIS, axon initial segment; SE, status epilepticus; DCX, doublecortin; AnkG, ankyrin G; DG, dentate gyrus; SRS, spontaneous recurrent seizures

Key words: temporal lobe epilepsy, neurogenesis, axon initial segment, neuronal plasticity, neuronal excitability

\section{Introduction}

Temporal lobe epilepsy (TLE) is characterized by the recurrence of seizure activity; however, the impact of frequent seizure activity on the neuronal networks of the brain is still not completely understood. Likewise, it is unclear why hippocampal newborn neurons after status epilepticus (SE) exhibit hyperexcitability in the hippocampal network and then influence ongoing epilepsy-related processes.

Seizure-induced increases in hippocampal neurogenesis have been observed in many TLE models, which are thought to contribute to abnormal brain activity (1). The newly-generated neurons originate primarily from neural progenitor cells in the subgranular zone of the dentate gyrus (DG) of hippocampus after epilepsy. They eventually integrate into hippocampal circuitry and may contribute to hippocampal network plasticity after the initial insult (2). These newly-generated neurons have immature synapses (3), which can receive synaptic input and transmit action potential output (4), and therefore become the center of spontaneous recurrent seizures (SRS). 5-bromo-2'-deoxyuridine (BrdU), an S-phase-specific marker, allows the detection of newly formed cells by incorporation into DNA (5). Because BrdU application is mainly conducted by intraperitoneal injection, epilepsy or ischemia which disturb or disrupt the function of the blood-brain barrier, might induce increases in the numbers of BrdU-labeled cells which is independent of changes in proliferation due to the altered BrdU availability in the brain $(6,7)$. Doublecortin (DCX), a protein associated with cell migration, which express in late type-3 progenitor cells in the process of neural stem cell proliferation and differentiation (8). DCX-positive cells are thought to be a migratory state as DCX plays a pivotal role during neural development where it is involved in radial glia-mediated neuronal migration and in hippocampal development (9). DCX-positive cells represent neural progenitor cell migration during hippocampal neurogenesis and the expression of DCX in newly-generated cells was increased after epilepsy stimuli (10). Thus, DCX marker was used in our study to identify the developmental stage of migration and differentiation of newborn neurons in hippocampus.

Axon initial segment (AIS) is an excitable neuronal domain positioned between the axonal and somato-dendritic compartment, which is involved in action potential initiation and 
fine-tunes the excitability of neurons (11). In recent years, our understanding of the molecular structure of AIS, its structural and functional plasticity related to the excitability of neurons, has seen major advances $(12,13)$. Ankyrin $G(A n k G)$ protein, a vital scaffolding molecule in AIS has been accepted and widely used as a biomarker to reflect the molecular structure of AIS (14). It is now widely accepted that the structural properties of AIS, such as length or location relative to the soma, change in an activity-dependent manner (15). This structural plasticity of AIS is known to be crucial for homeostatic control of neuronal excitability. The impact of AIS on neuronal excitability is dependent on the composition and characteristics of ion channels in the domain (16). In cultured hippocampus neurons, the location of AIS changes during the depolarization, but the movement of AIS can be prevented by the T/L-type calcium currents blocker Mibefradil (17). However, we still have little knowledge about how AIS plasticity in hippocampal newly-generated neurons altered after SE and its role in recurrent seizures. So far, the effects of calcium-current blocker Mibefradil on AIS plasticity and neuronal excitability in newly-generated neurons after epilepsy in vivo have not been determined.

In the present study, we first established pilocarpine-induced epileptic rats to assess hippocampal neurogenesis after pilocarpine-induced TLE and then explore AIS plasticity in hippocampal newly-generated neurons using immunofluorescent labeling of DCX and AnkG. Because voltage-gated calcium channels in AIS is essential for neuronal excitability, we suppose that used calcium channels blocker Mibefradil treatment could have effects on changes of AIS plasticity in hippocampal neurogenesis and neuronal excitability. Simultaneously, we employed electroencephalography (EEG) to detect the long-term consequences after treatment in pilocarpine-induced TLE rats. The ultimate goal of this study is to provide experimental evidence for AIS plasticity in hippocampal neurogenesis in the management of epileptic patients.

\section{Materials and methods}

Animals and pilocarpine-induced epilepsy models. Healthy, young male Sprague-Dawley (SD) rats (aged 6-8 weeks, 200-250 g, n=72) were used in this study. Rats were randomly assigned as control rats, rats subjected to pilocarpine, post-SE rats treated with Mibefradil and treated with Mibefradil only. Rats were injected intraperitoneally (i.p.) with lithium chloride (127 mg/kg; Sigma-Aldrich, St. Louis, MO, USA) and pilocarpine (25 mg/kg; Sigma-Aldrich). Racine's classification was used to evaluate seizure severity (18). After SE, all rats were administered with chloral hydrate injection $(3 \mathrm{ml} / \mathrm{kg}$, i.p.; Tonghua Dongbao Pharmaceutical Co., Ltd., Tonghua, China) to stop behavioral seizures. The matched control rats were injected i.p. with the same amount of normal saline. All procedures involving animals were performed according to protocols approved by the Institutional Animal Care and Use Committee of Central South University. The acute stage and chronic stage are period from 24 h to 7 days, 50-60 days post-SE, respectively. In our study, day 7 and day 60 were used to represent the corresponding acute and chronic stages. The same amounts of experimental and control rats were sacrificed at different time points, respectively, at day 7 and day $60(n=9$ in each group at each time point).

Implantation of the mini drug delivery system. Within $6 \mathrm{~h}$ after SE onset, the rats were anesthetized and fixed into the stereotaxic apparatus. Each mini-osmotic pump (ALZET ${ }^{\circledR}$ micro-osmotic pump, 1007D; Durect Corporation, Cupertino, CA, USA) was attached to a cannula and placed in a subcutaneous pocket. Subsequently, the cannula was implanted into the lateral hippocampus (positions: $-4.0 \mathrm{~mm}$ posterior to bregma, $3.0 \mathrm{~mm}$ bilateral to the midline, and $3.1 \mathrm{~mm}$ under the skull surface) with infusion of Mibefradil $(1.5 \mathrm{mg} / \mathrm{ml}$ in $0.1 \mathrm{M}$ PBS; Sigma-Aldrich) for seven consecutive days $(19,20)$. The cannula was secured with dental acrylic cement.

Behavioral recording. On day 60 post-SE, we recorded SRSs by using video monitoring for 14 days from 8:00-10:00 a.m. to 4:00-6:00 p.m. each day. The latency period (days), seizure frequency by cumulative analysis over time (per day) and seizure duration $(\mathrm{sec})$ were recorded. During this period, animals had free access to food and water as normal and no rats died. After behavioral observation, all the rats were sacrificed under anesthesia for the next experiments.

Implantation of EEG recording electrodes and EEG analysis. In the chronic stage of SE, animals were anesthetized using $10 \%$ chloral hydrate $(3 \mathrm{ml} / \mathrm{kg}$, i.p.), then fixed into the stereotaxic apparatus. Electrodes, consisting of a stainless steel wire were implanted into the positions: $2.5 \mathrm{~mm}$ anterior to bregma, $2.5 \mathrm{~mm}$ bilateral to midline, and $2.5 \mathrm{~mm}$ posterior to lambda and fixed with dental cement. After recovery, spontaneous EEG seizures were recorded for $2 \mathrm{~h}$ to quantify the frequency and mean duration during an EEG recording session. Data were collected and analyzed using an acquisition system (Physical Signal Recorder RM6240; Chengdu Instrument Factory, Chengdu, China). The EEG data were analyzed by an experienced neurologist.

Immunofluorescence staining. The rats were deeply anesthetized and transcardially perfused with $4 \%$ paraformaldehyde. The brains were excised and postfixed, then cut using a cryotome into serial coronal sections (25 $\mu \mathrm{m}$ thick) (Leica CM 1850; Leica, Wetzler, Germany). Sections were incubated in a blocking solution (10\% normal donkey serum, $0.3 \%$ Triton $\mathrm{X}-100$ in PBS, $2.5 \%$ bovine serum albumin in PBS) at room temperature for $2 \mathrm{~h}$. For DCX staining, the sections were washed and incubated for $12 \mathrm{~h}$ at $4^{\circ} \mathrm{C}$ with goat anti-DCX antibody (diluted 1:125; Santa Cruz Biotechnology, Inc., Santa Cruz, CA) followed by washing and incubation for $2 \mathrm{~h}$ in secondary antibody (diluted 1:1,000; Alexa Fluor 555 donkey anti-goat IgG; Invitrogen Life Technologies, Carlsbad, CA, USA). For AnkG/DCX double staining, sections were incubated in mouse anti-AnkG antibody (diluted 1:50; Merck Millipore, Darmstadt, Germany) and goat anti-DCX antibody (diluted 1:125; Santa Cruz Biotechnology, Inc.) at $4^{\circ} \mathrm{C}$ for $48 \mathrm{~h}$. Sections were rinsed and incubated with Alexa Fluor 488 donkey anti-mouse IgG + Alexa Fluor 555 donkey anti-goat IgG (all diluted 1:1,000; Invitrogen Life Technologies). The sections were then washed and mounted with Vectashield mounting media. 
Quantification. Immunopositive cells were counted by an experimenter blinded to the treatment conditions. The DG was operationally defined as a region extending from the interface of the hilus and granule cell layer to a width of approximately two cell bodies into hilus. The immunofluorescence images of DCX-positive cells in DG were acquired with a $20 \mathrm{X}$ objective (DM5000B light microscope; Leica). For quantification analysis, sections were coded and the captured images were analyzed using the ImageJ software package (National Institutes of Health, Bethesda, MD, USA). Five microscopic fields from the hippocampal DG regions were selected to calculate neuronal counts in each view at $x 40$ magnifications. The number of DCX immunopositive cells from five sections was counted and a mean cell count per section was obtained.

Dendrite length was detected using a confocal microscope (Carl Zeiss AG, Oberkochen, Germany) from five coronal sections per rat. Three-dimensional reconstructions of the DCX-positive cells were performed from Z-series stacks of confocal images. Each section was scanned as a $0.5 \mu \mathrm{m}$ thick Z-stack and included $30 \mathrm{Z}$ sections that contained the entire region of DCX-positive. DCX-labeled cells were then counted by analyzing each $\mathrm{Z}$ section and the reconstructed intact dendrite length were measured by reconstructed Z-sections projected with ImageJ software. The apical dendrite lengths of the DCX-positive cells were traced and analyzed. For AIS analysis, the lengths and position of AIS were measured from five coronal sections per rat with using ImageJ software package.

Statistics. All continuous variables were tested to confirm that they fit a normal distribution before further analysis. For analysis of DCX-positive cells and dendrite length, a 3-way ANOVAs followed by Tukey multiple comparison tests $(\alpha=0.05)$ was used to calculate the difference between any two groups of rats of different stages. For analysis of AIS changes per microscopic view, 3-way ANOVAs followed by Tukey multiple comparison tests $(\alpha=0.05)$ to was used to compare between-group difference in rats of different stages. For analysis of EEG data, one-factor ANOVA followed by Tukey multiple comparison tests $(\alpha=0.05)$ to was used to compare between-group difference in rats. Results are presented throughout as mean \pm standard deviation. $\mathrm{P}<0.05$ was considered to indicate a statistically significant difference. three-way ANOVAs were performed using SPSS 21.0 (SPSS, Inc, Chicago, IL, USA); all other statistical analyses were performed using GraphPad Prism 6.0 (GraphPad Software, Inc., La Jolla, CA, USA).

\section{Results}

Proliferation of newborn neurons in pilocarpine-induced epileptic rats. The birth of new neurons in DG area of hippocampus progresses from progenitor cells expressed DCX to mature neurons expressed the marker NeuN. Double immunofluorescence staining of DCX and NeuN were used to evaluate the newborn neurons in hippocampus of rats; typical images are showed in Fig. 1. Compared with controls, SE rats showed significantly increased number of newborn neurons in the DG of hippocampus on day 7 (ANOVA with post hoc Tukey's test, DCX/NeuN-positive cells: $220 \pm 13.9$ vs. $80.7 \pm 5.0, n=18$ per group, $\mathrm{P}<0.001)$. In epileptic rats on day 60 , the number of newborn neurons in the entire DG in SE rats appeared to decline compared to that of control rats (ANOVA with post hoc Tukey's test, DCX/NeuN-positive cells: $76.0 \pm 10.7$ vs. $66.4 \pm 6.5, \mathrm{n}=18$ per group, $\mathrm{P}=0.004$ ).

To clarify the function of AIS plasticity of newborn neurons in epileptogenesis, we used T/L-type calcium blocker Mibefradil treatment using intrahippocampal infusion. Quantitative analysis of the DCX staining was conducted to assess the neurogenesis in hippocampus, as showed in Fig. 1. Compared with Mibefradil-only rats, Mibefradil treatment after pilocarpine-induced epileptic rats showed DCX-positive cells increase in both days 7 and 60 (ANOVA with post hoc Tukey's test, $216.2 \pm 18.0$ vs. $79.5 \pm 11.7$ on day $7 ; 64.9 \pm 7.0$ vs. $78.9 \pm 9.6$ on day 60 , all $\mathrm{P}<0.001)$. However, no significant differences were observed for Mibefradil-only and control groups (ANOVA with post hoc Tukey's test, $79.5 \pm 11.7$ vs. $80.7 \pm 5.0$ on day 7 ; $78.9 \pm 9.6$ vs. $76.0 \pm 10.7$ on day $60, n=18$ per group, all $\mathrm{P}>0.05$ ), Mibefradil treatment after SE rats and epileptic rats on both day 7 and day $60(216.2 \pm 18.2$ vs. $220 \pm 13.9$ on day $7, P=0.71$; $64.9 \pm 7.0$ vs. $66.4 \pm 6.5$ on day $60, \mathrm{P}=0.95 ; \mathrm{n}=18$ per group).

Dendrite development of newborn neurons in pilocarpine-induced epileptic rats. Also, morphological features of DCX-immunolabeled cells in the DG from epileptic rats were different from those observed from controls. Compared to control rats, epileptic rats showed significantly increased apical dendrites lengths in newborn neurons on both days 7 and 60 (ANOVA with post hoc Tukey's test, $472.7 \pm 30.5$ vs. $779.7 \pm 25.7 \mu \mathrm{m}$ on day $7 ; 548.6 \pm 20.6$ vs. $817.1 \pm 24.9 \mu \mathrm{m}$ on day $60, \mathrm{n}=18$ per group, all $\mathrm{P}<0.001$ ) (Fig. 2), suggesting that pilocarpine-induced epileptic rats exhibit longer and extended apical dendrites in hippocampal newborn neurons. Furthermore, the increased hippocampal newborn neurons in acute stage post-SE in our study are consistent with the report that acute seizures mainly prompted the proliferation of cells expressing DCX (21). These results indicate that these newborn neurons with longer, extended apical dendrites could facilitate reception of more excessive, recurrent synaptic input and then contribute to an increase in the excitability of hippocampal network after seizures.

Compared with Mibefradil-only rats, Mibefradil treatment rats showed significantly increased in the lengths of apical dendrites in newborn neurons on both days 7 and 60 (ANOVA with post hoc Tukey's test, $758.6 \pm 35.0$ vs. $458.0 \pm 25.9 \mu \mathrm{m}$ on day $7,806.6 \pm 27.6$ vs. $534.1 \pm 32.6 \mu \mathrm{m}$ on day $60, \mathrm{n}=18$ per group all $\mathrm{P}<0.001$ ) (Fig. 2). However, no significant differences in the apical dendrites lengths were found in newborn neurons between the Mibefradil treatment rats and epileptic rats (ANOVA with post hoc Tukey's test, $758.6 \pm 35.0$ vs. $779.7 \pm 25.7 \mu \mathrm{m}$ on day $7, \mathrm{P}=0.12 ; 806.6 \pm 27.6$ vs. $817.1 \pm 24.9 \mu \mathrm{m}$ on day 60 , $\mathrm{P}=0.61 ; \mathrm{n}=18$ per group), Mibefradil-only and control groups $(458.0 \pm 25.9$ vs. $472.7 \pm 30.5 \mu \mathrm{m}$ on day $7, \mathrm{P}=0.40 ; 534.1 \pm 32.6$ vs. $548.6 \pm 20.6 \mu \mathrm{m}$ on day $60, \mathrm{P}=0.33 ; \mathrm{n}=18$ per group). These results indicate that Mibefradil had less effect on the newborn neurons number and morphology in epileptic rats.

AIS plasticity of hippocampal newborn neurons in pilocarpine-induced epileptic rats. Double-immunofluorescence method with AnkG and DCX was used to reveal structural 

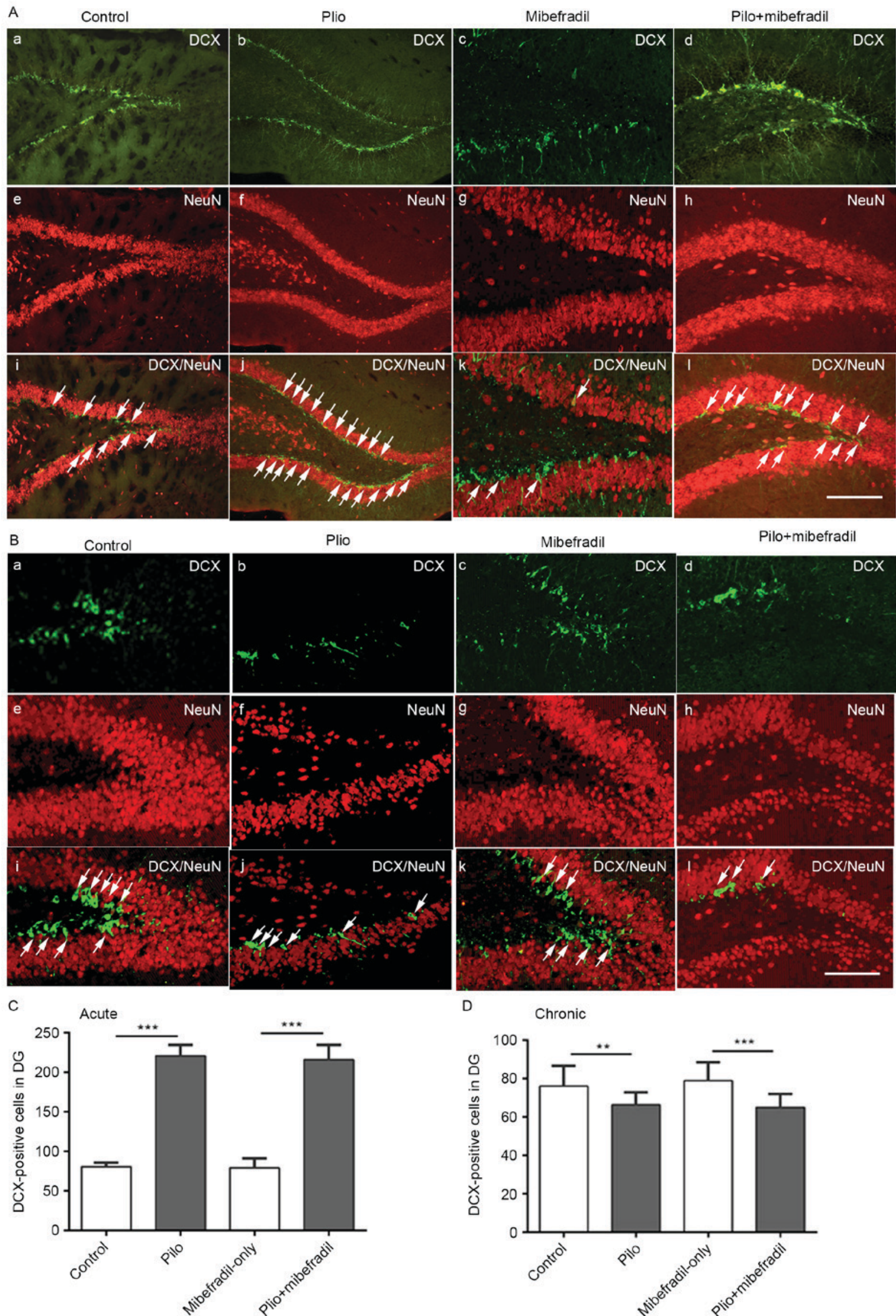

Mibefradil

Pilo+mibefradil

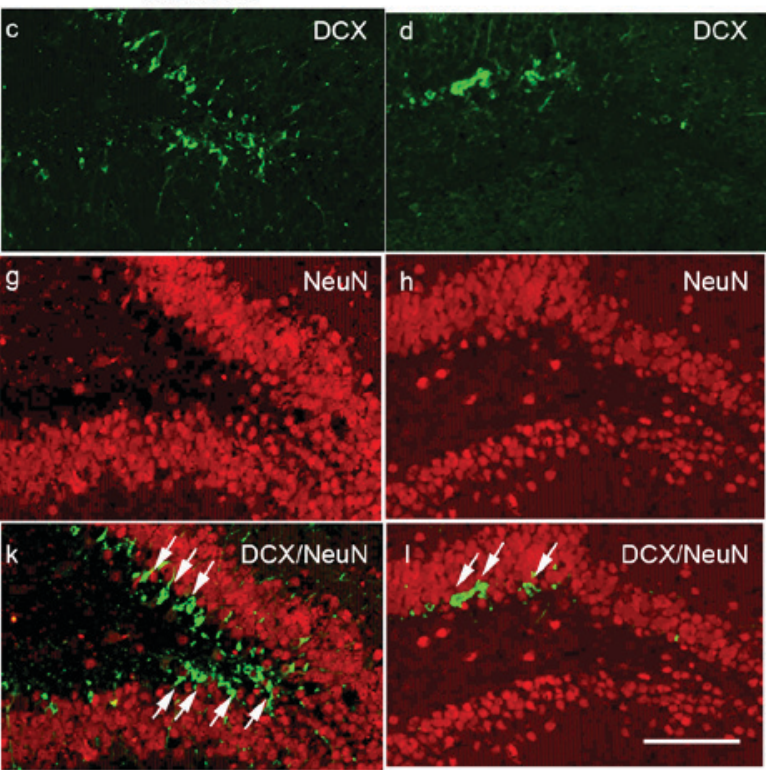

D Chronic

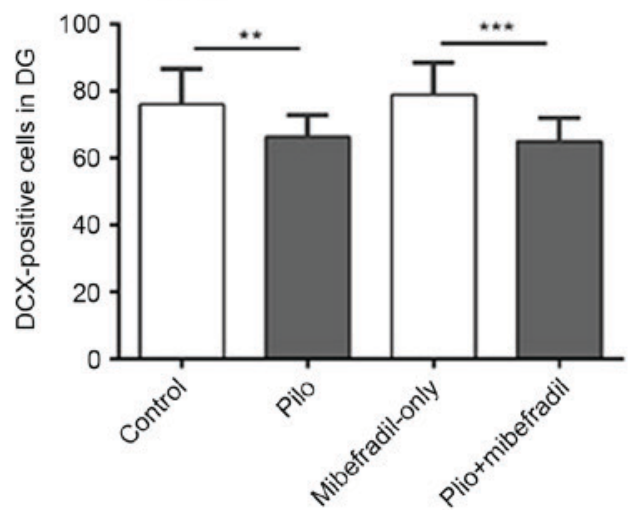

Figure 1. The proliferation of newborn neurons in the dentate gyrus (DG) area in hippocampus of different groups. Representative pictures of doublecortin (DCX)-positive cells in the hippocampus on days (A) 7 and (B) 60 and quantification of DCX-positive cells in hippocampus on days (C) 7 and (D) 60 . Arrows indicate the presence of DCX-positive cells. The proliferation of newborn neurons increased post-status epilepticus in DG compared to the control group and Mibefradil-only group $\left({ }^{* * * *} \mathrm{P}<0.01\right.$ vs. Control and Mibefradil-only; ${ }^{* *} \mathrm{P}<0.01$ vs. Control in chronic). However, no significant changes were observed in the DG region between the Pilo and the Mibefradil treatment groups (P>0.05 vs. Pilo). Scale bar indicates $100 \mu \mathrm{m}$. 
A

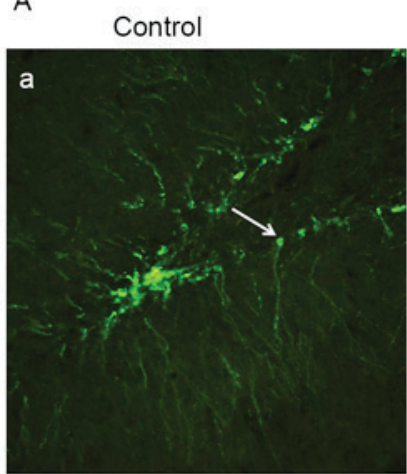

B

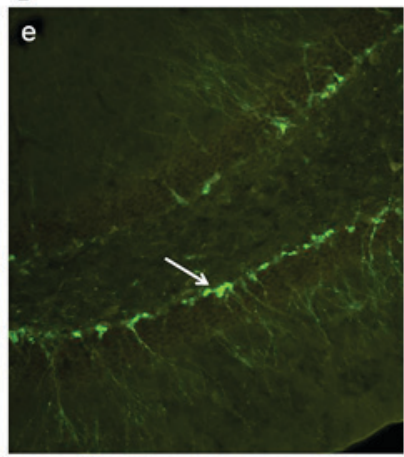

c

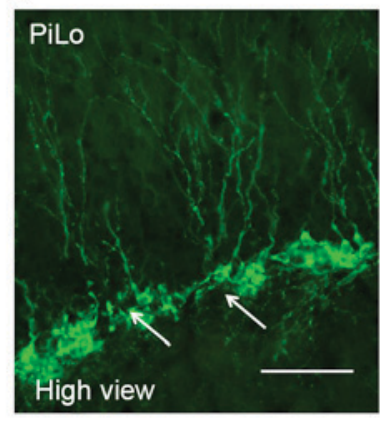

PiLo
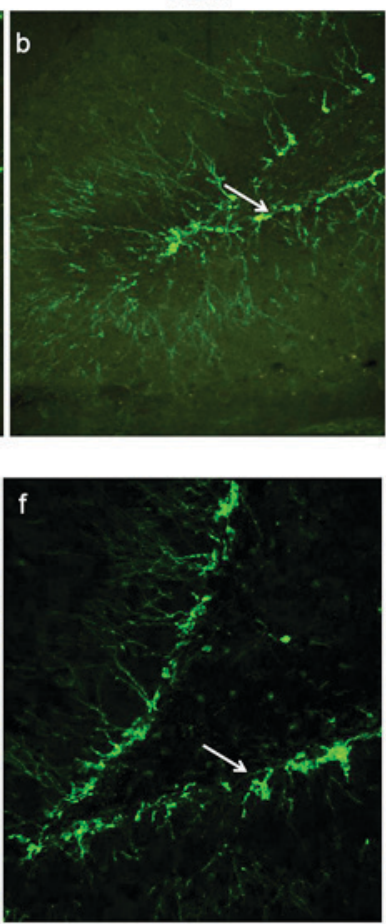

D

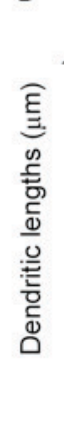

Acute
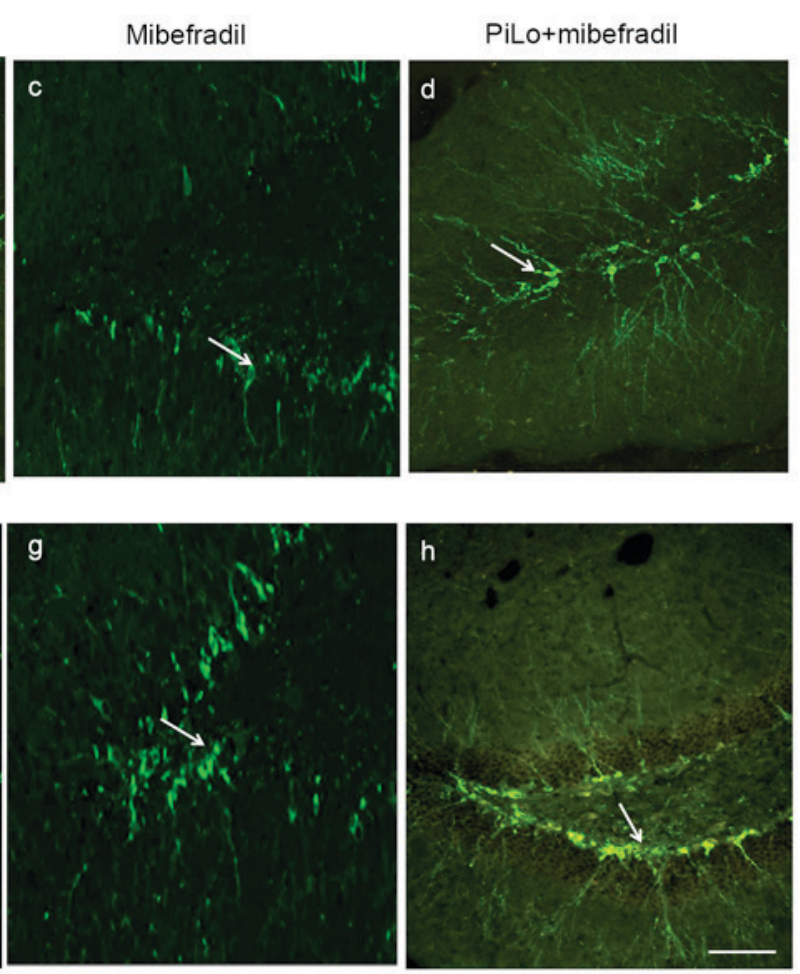

E

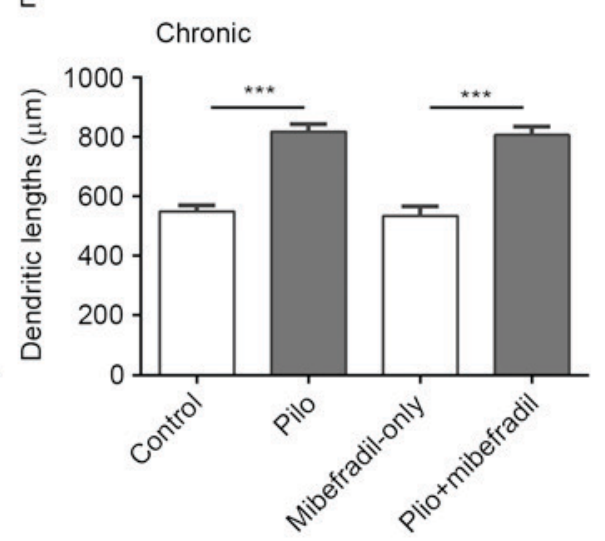

Figure 2. Dendrite development of newborn neurons in the dentate gyrus (DG) of hippocampus in different groups. Representative images of doublecortin (DCX)-positive cells showed the dendrite development of newborn neurons in the DG (A) post 7 days after status epilepticus (SE) and (B) 60 days after SE (white arrows). The quantifications of the length of dendrites in acute and chronic stages were showed in graphs D and E. The length of dendrite in newborn neurons increased post-SE in DG compared to the control group and Mibefradil-only group $\left({ }^{* * *} \mathrm{P}<0.01\right.$ vs. Control; ${ }^{* * *} \mathrm{P}<0.01$ vs. Mibefradil-only). However, no significant changes were observed in the DG region between the Pilo and the Mibefradil groups (P>0.05 vs. Pilo). (A and B) Scale bars indicate $50 \mu \mathrm{m}$ and (C) $20 \mu \mathrm{m}$.

plasticity of the AIS in newborn neurons in epileptic rats; representative images are showed in Figs. 3 and 4. Compared with the control rats, rats subjected to pilocarpine showed reduced lengths of AIS in newborn neurons of hippocampus on both days 7 and 60 (ANOVA with post hoc Tukey's test, $29.6 \pm 0.7$ vs. $25.3 \pm 0.9 \mu \mathrm{m}$ on day $7 ; 29.9 \pm 0.7$ vs. $26.1 \pm 1.0 \mu \mathrm{m}$ on day $60, \mathrm{n}=18$ per group, all $\mathrm{P}<0.001$ ). To further analyze the position of AIS in newborn neuron, we measured the distance of AIS from the soma. As showed in Figs. 3A and $4 \mathrm{~A}$, the distance of AIS from the soma was significantly decreased in epileptic rats compared to the control rats in both days 7 and 60 (ANOVA with post hoc Tukey's test, $2.6 \pm 0.3$ vs. $1.7 \pm 0.3 \mu \mathrm{m}$ on day $7 ; 2.6 \pm 0.3$ vs. $1.9 \pm 0.2 \mu \mathrm{m}$ on day $60, n=18$ per group, all $\mathrm{P}<0.001)$. All results indicate that the plasticity of AIS, reflecting on the decreases in the length of AIS and shortening of the position of AIS from soma during both acute and chronic status epileptics, is probably triggered by the altered environment induced by epileptic seizures.

Interestingly, Mibefradil treatment resulted in increases in the lengths of AIS in newborn neurons compared with the epileptic controls on both post-SE days 7 and 60, (ANOVA with post hoc Tukey's test, $28.3 \pm 0.59$ vs. $25.3 \pm 0.95 \mu \mathrm{m}$ on day $7 ; 28.6 \pm 0.93$ vs. $26.1 \pm 0.95 \mu \mathrm{m}$ on day $60, \mathrm{n}=18$, all $\mathrm{P}<0.001)$. Comparisons of AIS location from the soma in newborn neurons showed greater distance increases in the epileptic rats treated with Mibefradil on both post-SE days 7 and 60 than epileptic rats (ANOVA with post hoc Tukey's test, $2.24 \pm 0.38$ vs. $1.71 \pm 0.30 \mu \mathrm{m}$ on day $7 ; 2.41 \pm 0.46$ vs. $1.90 \pm 0.19 \mu \mathrm{m}$ on day $60, \mathrm{n}=18$ per group, all $\mathrm{P}<0.001$ ) 
A
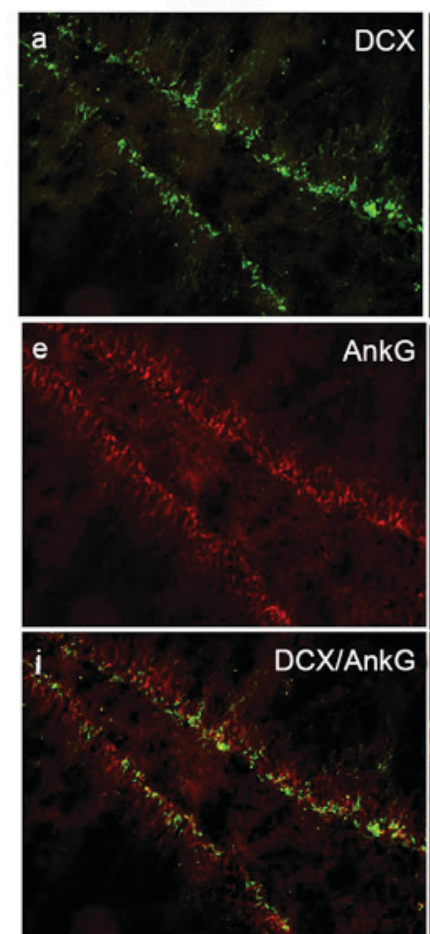

B

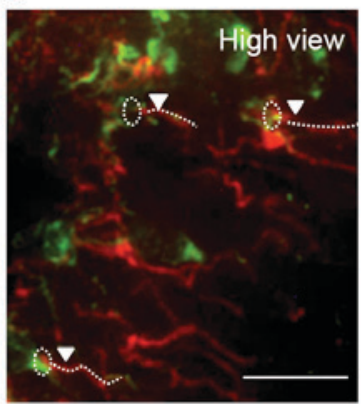

C
Pilo
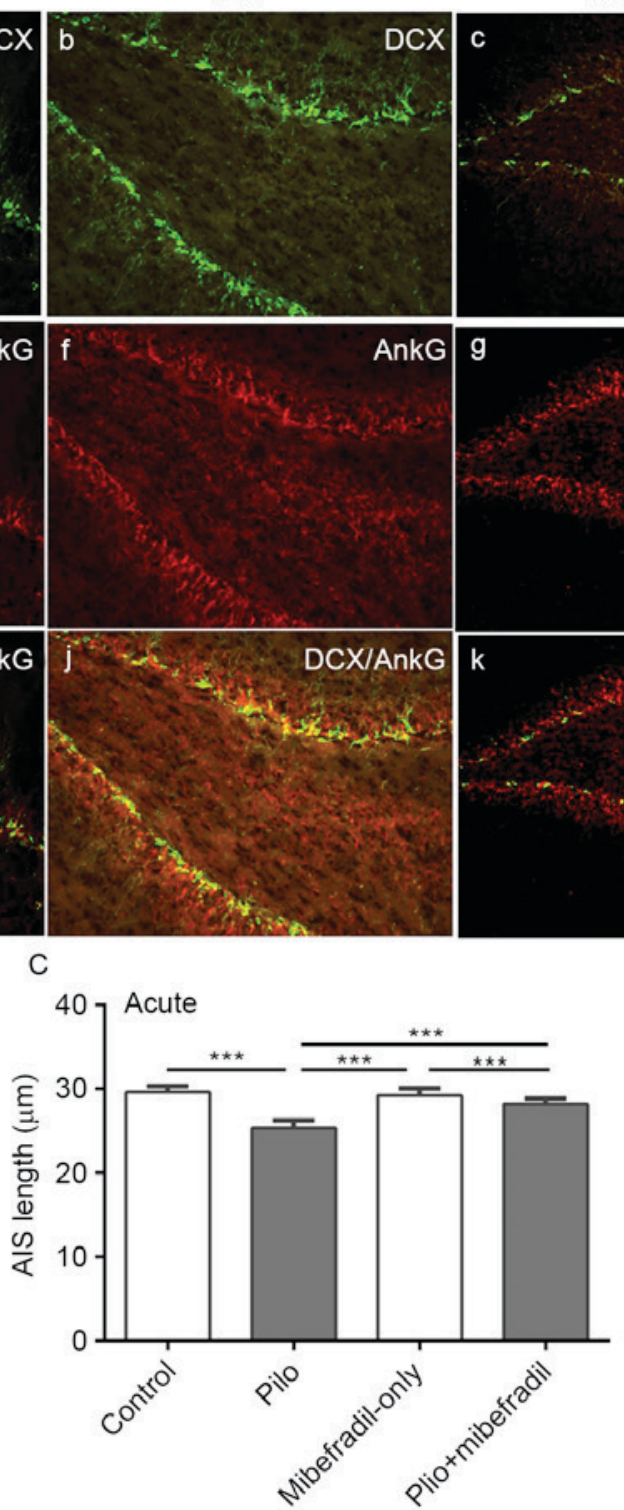

Mibefradil
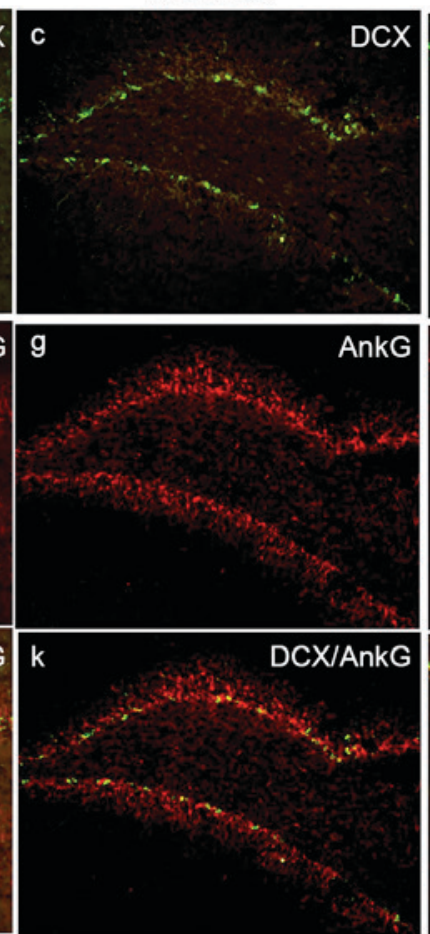

$D$

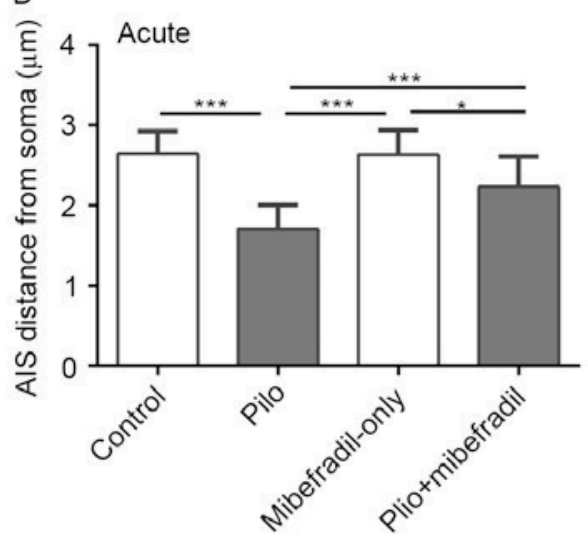

Figure 3. The movement of axon initial segment (AIS) of newborn neurons in the dentate gyrus (DG) in the acute stage after status epilepticus (SE). Representative pictures of AIS movement in (A and B) doublecortin (DCX)-positive cell and (C and D) the quantification of the length of AIS and distance of AIS from soma in acute stage. As showed in the graphs, Mibefradil treatment suppressed the movement of AIS post-SE in acute stage $\left({ }^{* * *} \mathrm{P}<0.01\right.$ vs. Control and Mibefradil-only; ${ }^{*} \mathrm{P}<0.01$ vs. Mibefradil-only in AIS distance). Scale bars indicate $50 \mu \mathrm{m}$ (A) and $20 \mu \mathrm{m}$ (B).

(Figs. 3A and 4). However, Mibefradil treatment showed significantly differences in both length and distance of AIS in newborn neurons compared with Mibefradil-only group (length: $29.3 \pm 0.79$ vs. $28.3 \pm 0.59 \mu \mathrm{m}$ on day $7, \mathrm{P}=0.003$; $29.3 \pm 0.80$ vs. $28.6 \pm 0.93 \mu \mathrm{m}$ on day $60, \mathrm{P}=0.02$; distance: $2.64 \pm 0.30$ vs. $2.24 \pm 0.38 \mu \mathrm{m}$ on day $7, \mathrm{P}<0.001 ; 2.74 \pm 0.31$ vs. $2.41 \pm 0.46 \mu \mathrm{m}$ on day $60, \mathrm{P}=0.02 ; \mathrm{n}=18$ per group), suggesting of no notable AIS changes in Mibefradil-only rats. These results indicated that Mibefradil treatment can effectively reverse the movement of AIS post-SE, though the changes in aberrant neurogenesis and dendrites were not seen.

The effect of mibefradil on behavioral outcomes and spontaneous EEG seizures in chronic TLE. In the present study, pilocarpine-induced seizures were characterized by brief tonic-clonic episodes with forelimb automatisms with rearing and falling, which is similar to the descriptions from previous reports (22). No seizures were observed in control and Mibefradil-only groups. Data in Fig. 5E concerning the behaviors showed that Mibefradil treatment substantially shortened the mean duration of spontaneous seizures compared with the pilocarpine group (ANOVA with post hoc Tukey's test, $26.5 \pm 1.9$ vs. $39.0 \pm 1.8 \mathrm{~s}, \mathrm{n}=3$ per group, $\mathrm{P}<0.001$ ). In addition, the average latency periods were longer and the frequency of spontaneous seizures was significantly reduced in epileptic rats with Mibefradil treatment compared with the epileptic controls (ANOVA with post hoc Tukey's test, $12.5 \pm 1.9$ vs. $6.3 \pm 1.4$ days, $\mathrm{n}=3$ per group, $\mathrm{P}<0.001$ ) (Fig. $5 \mathrm{~F}$ and $\mathrm{G}$ ).

The spontaneous seizures measured by EEG recordings showed that epileptic rats promoted the development of SRS with characteristic spike and wave activity of EEG in the chronic phase of TLE. No EEG seizures were observed in 
A Control

Pilo Mibefradil Pilo+mibefradil
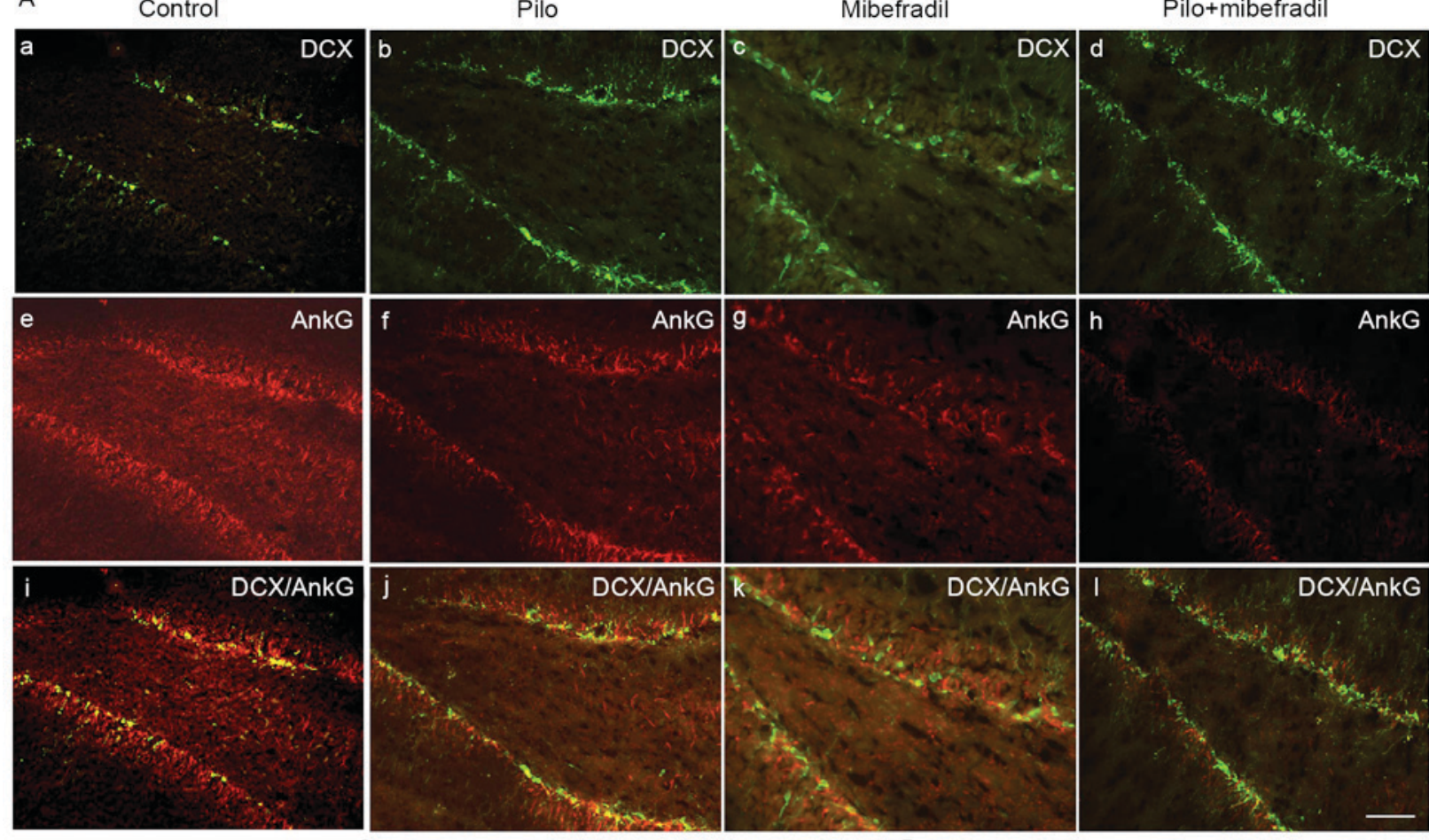

B

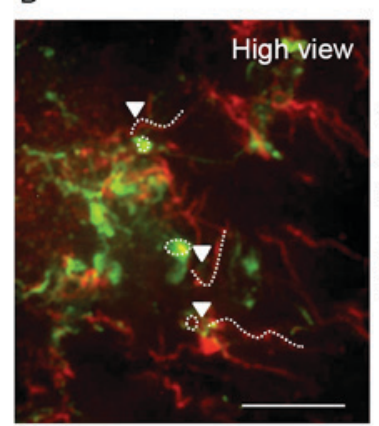

C

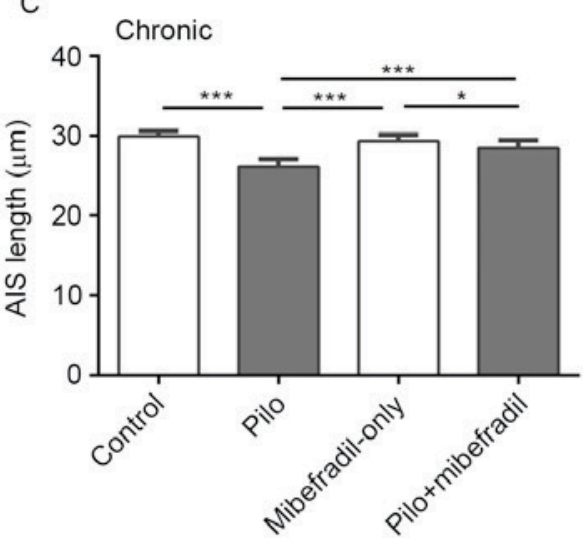

D

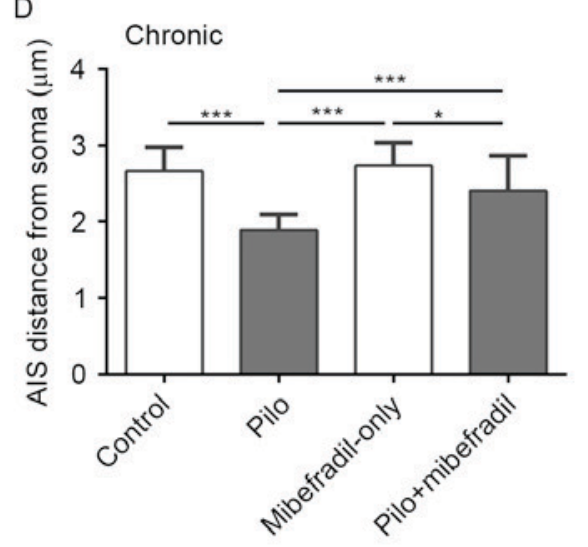

Figure 4. The movement of axon initial segment (AIS) of newborn neurons in the DG in the chronic stage after status epilepticus (SE). Representative pictures of AIS movement in (A and B) doublecortin (DCX)-positive cell and (C and D) the quantification of the length of AIS and distance of AIS from soma. Mibefradil treatment suppressed the movement of AIS post-SE in chronic stage $\left({ }^{* * * *} \mathrm{P}<0.01\right.$ vs. Control; ${ }^{* * * *} \mathrm{P}<0.01$ vs. Pilo; ${ }^{*} \mathrm{P}<0.01$ vs. Mibefradil-only). Scale bars indicate (A) $50 \mu \mathrm{m}$ and (B) $20 \mu \mathrm{m}$.

control rats and rats receiving Mibefradil-only. Spontaneous EEG seizures were frequently observed in rats subjected to pilocarpine; typical EEG images are showed in Fig. 4A. During the $2 \mathrm{~h}$ EEG recording, Mibefradil treatment rats showed significantly reduced firing rates and shortened the mean duration of spontaneous seizures compared with the pilocarpine rats (ANOVA with post hoc Tukey's test, firing rates: $29.9 \pm 1.4$ vs. $37.3 \pm 1.2 \mathrm{~Hz}, \mathrm{P}<0.001$; duration: $2.4 \pm 0.19$ vs. $5.4 \pm 0.19 \mathrm{sec}, \mathrm{P}<0.001 ; \mathrm{n}=3$ per group) (Fig. $5 \mathrm{~B}$ and $\mathrm{C}$ ). In addition, compared with the epileptic rats, epileptic rats with Mibefradil treatment showed reduced frequency of spontaneous EEG seizures (ANOVA with post hoc Tukey's test, 25.5 \pm 2.4 vs. 34.7 $\pm 3.1, \mathrm{n}=3, \mathrm{P}<0.001$ ) (Fig. 5D). All results indicate that Mibefradil treatment rescued the AIS movement and alleviated the spontaneous seizures in epileptic rats, suggesting that Mibefradil treatment can fine-tune the neuron excitability and firing behavior after epilepsy.

\section{Discussion}

In the present study, we investigated features of hippocampal neurogenesis and AIS plasticity in newborn neurons in both acute and chronic phases of the experimental epileptic rats. The pilocarpine-induced rats of TLE resulted in increased proliferation and abnormal dendrite development of newborn neurons. More importantly, we found a significant decrease in AIS length and distance in hippocampal neurogenesis in pilocarpine-induced epileptic rats, with increased spontaneous seizures observed in both behavioral and EEG recordings. After Mibefradil treatments, AIS length and distance tend to increase in epileptic rats, but no significant changes were observed in Mibefradil-only rats. By comparing the EEG and behaviors in Mibefradil treatment rats and epileptic rats, Mibefradil treatment rats were distinguished from non-treatment rats with decreased seizure activity. 

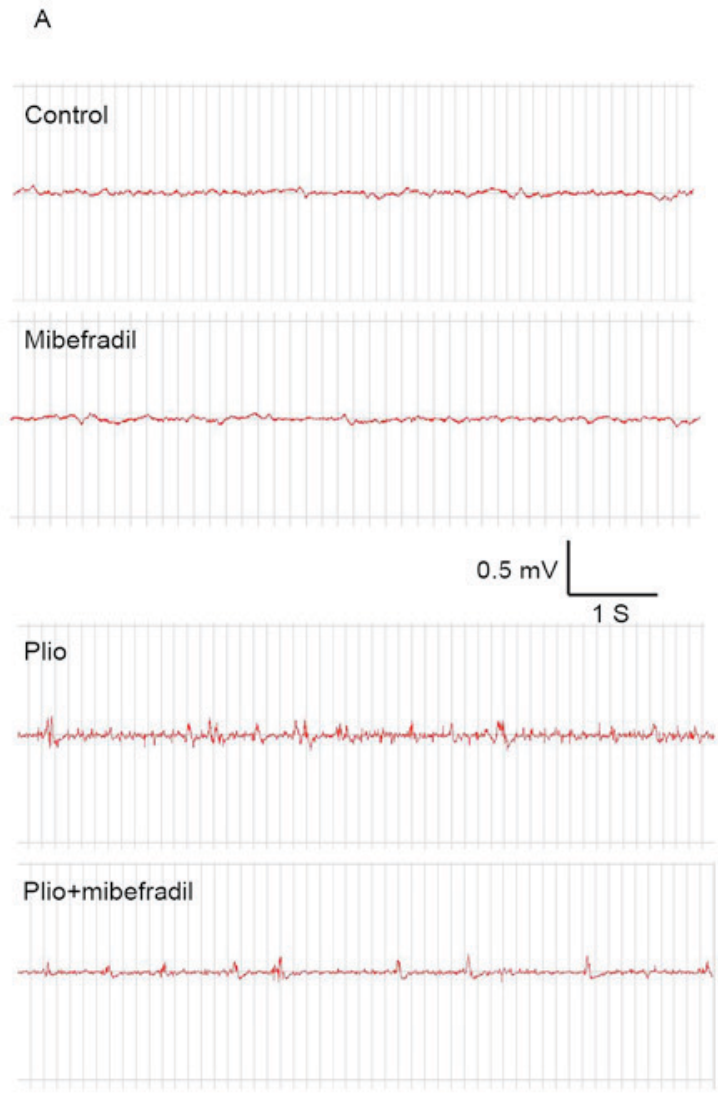

B
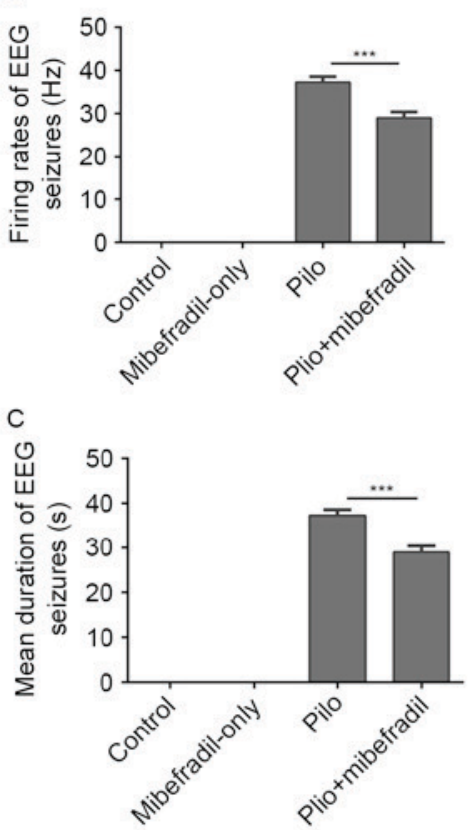

D

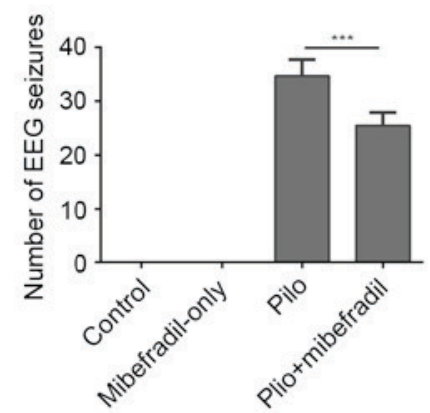

$\mathrm{E}$

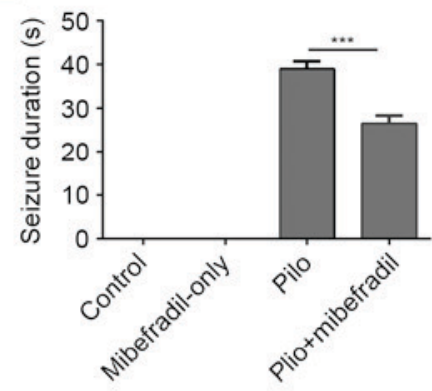

$\mathrm{F}$

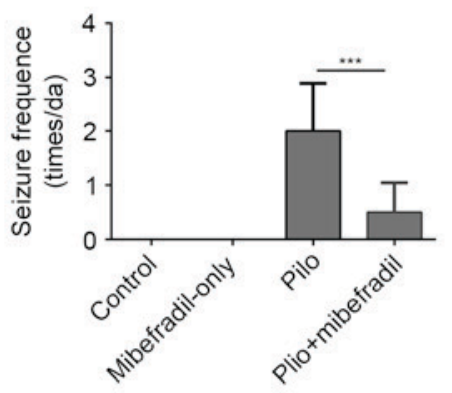

G

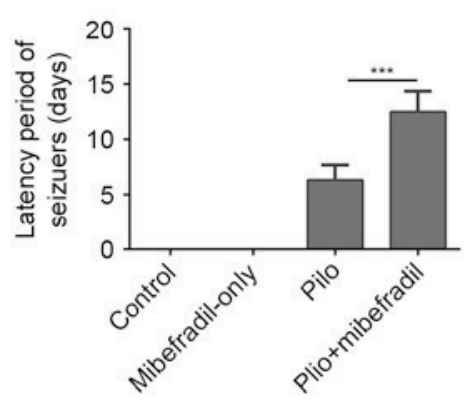

Figure 5. Spontaneous electroencephalography (EEG) seizures and behaviors in the chronic stage of temporal lobe epilepsy. Examples of rhythmic epilepticform activity of different groups (A). EEG results showed the seizures EEG post-status epilepticus (SE), while no seizures were found in control and Mibefradil only groups. Graphs highlight the firing rates of EEG seizures (B), mean durations of spontaneous EEG seizures (C) and frequency of spontaneous EEG seizures (D). In behavioral records, the mean durations $(E)$, average frequency $(F)$ and latency $(G)$ of spontaneous seizures presented similar changes as EEG results showed. Mibefradil treatment can suppress the spontaneous seizures post-SE in chronic stage ( ${ }^{* * * *} \mathrm{P}<0.01$ vs. Pilo).

These results suggest that the AIS plasticity in hippocampal newly-generated neurons is associated with hyperexcitability of newborn neurons in pilocarpine-induced rats of TLE. To our knowledge, this is the first study that details the changes of AIS movement in hippocampal neurogenesis in epilepsy and explores their relationship with the development of recurrent seizures.

Growing evidence has showed that epileptic animals often experience the proliferation and differentiation of the neural progenitor cells in the DG area of hippocampus, as well as changes of neuronal excitability $(23,24)$. This dramatic increase in the production of new neurons in the DG following SE can contribute to, rather than counteract, abnormal brain activity $(1,25)$. These newly-generated neurons developed abnormal dendrites which can facilitate reception of more excessive, recurrent synaptic input by aberrantly sprouted mossy fibers, and then contribute to an increase in the excitability of the hippocampal network after seizures $(26,27)$. Elucidation of this hyperexcitability of hippocampal network can facilitate the SRS mechanism in TLE. In this study, epileptic rats showed increased hippocampal neurogenesis and abnormal dendrite development of newborn neurons in the acute stage of epileptic models and subsequent reduction in the chronic stage of TLE. Also, seizures were always observed in chronic stage of both epileptic patients and animals. These results suggested that changes in hippocampal newly-generated neurons may be closely related to the increased neuronal excitability in the recurrence of seizure activity in the TLE. Our results are consistent with early studies showing abnormal hippocampal neurogenesis in epileptic rats (28); however, the find that newborn neurons plasticity and excitability has not been reported previously.

Besides detailing changes in newborn neurons in hippocampus, we also explored the changes of neuronal excitability domain, AIS in hippocampal new-generated neurons. AIS is a specialized domain of the axon proximal to the soma and can play a major role in neuronal excitability $(29,30)$. It is widely accepted that the structural characteristics of the AIS such as length and position relative to soma, can contribute to homeostatic control of excitability (31). This structural plasticity of AIS may regulate the excitability of a neuron in response to its environment, either attenuating or enhancing 
a neuron's sensitivity $(32,33)$. Our study revealed TLE rats showed decreased length and distance to the soma in AIS of hippocampal newborn neurons in both acute and chronic stages post-SE. These findings are consistent with previous study of AIS in motor-sensory cortical neurons in malformations of cortical development and epileptic rats (34). However, it must be noted that AIS plasticity in this study was from hippocampal newborn neurons in epileptic rats in which AIS length and distance were notably decreased compared with controls. Thus, it is required to learn the hippocampal newborn neurons when study the mechanism of epilepsy.

There are several causes explaining the changes of AIS plasticity observed in epileptic rats. The initial decrease in AIS length of hippocampal newborn neurons could be triggered by the proteolysis of the AnkG introduced by calcium-dependent cysteine protease calpain in the context of injury (35). Because the proteolysis of AIS cytoskeletal proteins are rapid and irreversible (36), the AIS cytoskeleton disrupts as we observed in epileptic rats in both acute and chronic stages. For another, change in AIS location is related to the extent of its isolation from the soma and a greater distance can increase the isolation (29). When the AIS is located proximally from the soma, the AIS becomes more excitable because the charge dissipation decreases, making it easy to depolarize the AIS above the action potential threshold in the presence of the shunting conductance. Such a finding is consistent with the reports by Harty et al (13), who showed that AIS is closer to the soma in pyramidal neurons in deep layer 5 of somatosensory cortex in amygdala kindling epileptic rats.

However, the changes of AIS plasticity in both length and distance as we observed in epileptic rats can mutually influence the neuronal excitability. For example, Baranauskas et al examined the AIS plasticity in cortical pyramidal neurons, finding that AIS length and location depend on the balance of its depolarizing accessibility and electrical isolation from somatodendritic conductance loads (37). Also, Gulledge and Bravo reported when AIS was shorter or adjacent to the soma in the dentate granule neuron, neuronal excitability was highest (38). Because ion channels in the AIS arrangement play distinct roles in action potential initiation, AIS plasticity may primarily through changes in AIS length to fine-tune excitability at relatively slow timescales after stimuli (39). Our study also revealed that the pilocapine-induced epileptic rats showed significantly decreased AIS lengths but only within a few micrometers in AIS locations.

AIS is enriched with multiple types of ion channels, including $\mathrm{Na}^{+}, \mathrm{K}^{+}$and more important, $\mathrm{Ca}^{+}$channels. The $\mathrm{Ca}+$ channels in AIS are essential for action potential initiation (40); thus it is practical to explore the calcium-current changes in AIS using the calcium-current blocker. Consistent with our findings, another study also showed a blocker Mibefradil could effectively suppress the AIS movement in hippocampal neurons in vitro (17), and imply that AIS plasticity in hippocampal newly-generated neuron can be a target excitable site for recurrent of epilepsy. However, our study also revealed the epilepsy network and long-term seizure activity attenuated in epileptic rats after Mibefradil treatment, supporting the contribution of AIS to neuronal excitability in epilepsy. These results suggested that AIS plasticity of newborn neurons could increase neuronal excitability after pilocarpine-induced
TLE rats, which may contribute to chronic epilepsy; this is congruent between in vitro and in vivo and provides the basis for studying the AIS plasticity occurring in human using animals.

We acknowledge that this study has some limitations. First, we did not perform electrophysiological studies on hippocampal newborn neurons in vitro to further verify the changes in AIS plasticity on newly-generated neurons. Further studies will need to clarify the details of changes in ion channels at the AIS such as using a novel method of axon-bleb patch-clamp together with somatic nucleated patch recording from hippocampal newborn neurons. Second, we cannot rule out the possibility that the treatment of Mibefradil can also affect other types of neurons other than newborn neurons. Moreover, not only the T/L-type calcium blocker Mibefradil, but also the L-type calcium currents blocker nifedipine can abolish activity-dependent AIS movement (17). It may require more specific research methods to distinguish the difference between the two types of calcium currents on AIS plasticity. Finally, it will be helpful to attempt other genetic engineering methods to explore possible related micro RNAs to further regulate the AIS expression and movement in epileptic animal or cultured hippocampal newborn neurons and then explore the pathophysiological mechanisms of the AIS plasticity in epilepsy.

In conclusion, our study confirmed the aberrant hippocampal neurogenesis together with prolonged plasticity of AIS in hippocampal newborn neurons in pilocarpine-induced TLE rats. Mibefradil treatment effectively suppressed the movement of AIS in hippocampal newborn neurons which was accompanied by decreased long-term seizure activity. These new findings have undoubtedly provided experimental evidence that AIS plasticity in newborn neurons play a key role in epilepsy and this might be a potential therapeutic target to help control seizure activities.

\section{Acknowledgements}

This study was supported by National Natural Science Foundation of China (nos. 81100967 and 81371435).

\section{References}

1. Murphy BL, Hofacer RD, Faulkner CN, Loepke AW and Danzer SC: Abnormalities of granule cell dendritic structure are a prominent feature of the intrahippocampal kainic acid model of epilepsy despite reduced postinjury neurogenesis. Epilepsia 53: 908-921, 2012.

2. Overstreet-Wadiche LS, Bromberg DA, Bensen AL and Westbrook GL: Seizures accelerate functional integration of adult-generated granule cells. J Neurosci 26: 4095-4103, 2006.

3. Shapiro LA and Ribak CE: Newly born dentate granule neurons after pilocarpine-induced epilepsy have hilar basal dendrites with immature synapses. Epilepsy Res 69: 53-66, 2006.

4. Wang LP, Kempermann G and Kettenmann H: A subpopulation of precursor cells in the mouse dentate gyrus receives synaptic GABAergic input. Mol Cell Neurosci 29: 181-189, 2005.

5. Bonzano S and De Marchis S: Detecting neuronal differentiation markers in newborn cells of the adult brain. Methods Mol Biol 1560: 163-177, 2017.

6. Erdő F, Denes L and de Lange E: Age-associated physiological and pathological changes at the blood-brain barrier: A review. J Cereb Blood Flow Metab 37: 4-24, 2017.

7. von Bohlen und Halbach O: Immunohistological markers for proliferative events, gliogenesis, and neurogenesis within the adult hippocampus. Cell Tissue Res 345: 1-19, 2011. 
8. Jessberger S, Römer B, Babu H and Kempermann G: Seizures induce proliferation and dispersion of doublecortin-positive hippocampal progenitor cells. Exp Neurol 196: 342-351, 2005.

9. Corbo JC, Deuel TA, Long JM, LaPorte P, Tsai E, Wynshaw-Boris A and Walsh CA: Doublecortin is required in mice for lamination of the hippocampus but not the neocortex. J Neurosci 22: 7548-7557, 2002.

10. Kim JH, Jang BG, Choi BY, Kwon LM, Sohn M, Song HK and Suh SW: Zinc chelation reduces hippocampal neurogenesis after pilocarpine-induced seizure. PLoS One 7: e48543, 2012.

11. Petersen AV, Cotel F and Perrier JF: Plasticity of the axon initial segment: Fast and slow processes with multiple functional roles. Neuroscientist: May 3, 2016 (Epub ahead of print).

12. Hamada MS and Kole MH: Myelin loss and axonal ion channe adaptations associated with gray matter neuronal hyperexcitability. J Neurosci 35: 7272-7286, 2015.

13. Harty RC, Kim TH, Thomas EA, Cardamone L, Jones NC Petrou S and Wimmer VC: Axon initial segment structural plasticity in animal models of genetic and acquired epilepsy. Epilepsy Res 105: 272-279, 2013.

14. Hedstrom KL, Ogawa Y and Rasband MN: AnkyrinG is required for maintenance of the axon initial segment and neuronal polarity. J Cell Biol 183: 635-640, 2008.

15. Evans MD, Dumitrescu AS, Kruijssen DL, Taylor SE and Grubb MS: Rapid modulation of axon initial segment length influences repetitive spike firing. Cell Rep 13: 1233-1245, 2015.

16. Kole MH and Stuart GJ: Signal processing in the axon initial segment. Neuron 73: 235-247, 2012.

17. Grubb MS and Burrone J: Activity-dependent relocation of the axon initial segment fine-tunes neuronal excitability. Nature 465: 1070-1074, 2010

18. Racine RJ: Modification of seizure activity by electrical stimulation: Cortical areas. Electroencephalogr Clin Neurophysiol 38 $1-12,1975$

19. Chen YL, Tsaur ML, Wang SW, Wang TY, Hung YC, Lin CS, Chang YF, Wang YC, Shiue SJ and Cheng JK: Chronic intrathecal infusion of mibefradil, ethosuximide and nickel attenuates nerve ligation-induced pain in rats. Br J Anaesth 115: 105-111, 2015.

20. Lee TS, Kaku T, Takebayashi S, Uchino T, Miyamoto S, Hadama T, Perez-Reyes E and Ono K: Actions of mibefradil, efonidipine and nifedipine block of recombinant T- and L-type Ca channels with distinct inhibitory mechanisms. Pharmacology 78 11-20, 2006.

21. Parent JM, Tada E, Fike JR and Lowenstein DH: Inhibition of dentate granule cell neurogenesis with brain irradiation does not prevent seizure-induced mossy fiber synaptic reorganization in the rat. J Neurosci 19: 4508-4519, 1999.

22. Alam AM and Starr MS: Regional changes in brain dopamine utilization during status epilepticus in the rat induced by systemic pilocarpine and intrahippocampal carbachol. Neuropharmacology 35: 159-167, 1996.

23. Shu Y, Xiao B, Wu Q, Liu T, Du Y, Tang H, Chen S, Feng L, Long L and Li Y: The Ephrin-A5/EphA4 interaction modulates neurogenesis and angiogenesis by the p-Akt and p-ERK pathways in a mouse model of TLE. Mol Neurobiol 53: 561-576, 2016.
24. Bender RA, Dubé C, Gonzalez-Vega R, Mina EW and Baram TZ: Mossy fiber plasticity and enhanced hippocampal excitability, without hippocampal cell loss or altered neurogenesis, in an animal model of prolonged febrile seizures. Hippocampus 13 : 399-412, 2003

25. Parent JM, Yu TW, Leibowitz RT, Geschwind DH, Sloviter RS and Lowenstein DH: Dentate granule cell neurogenesis is increased by seizures and contributes to aberrant network reorganization in the adult rat hippocampus. J Neurosci 17: 3727-3738, 1997.

26. Kron MM, Zhang H and Parent JM: The developmental stage of dentate granule cells dictates their contribution to seizure-induced plasticity. J Neurosci 30: 2051-2059, 2010.

27. Ribak CE, Tran PH, Spigelman I, Okazaki MM and Nadler JV: Status epilepticus-induced hilar basal dendrites on rodent granule cells contribute to recurrent excitatory circuitry. J Comp Neurol 428: 240-253, 2000

28. Song $\mathrm{C}, \mathrm{Xu} \mathrm{W}$, Zhang $\mathrm{X}$, Wang $\mathrm{S}$, Zhu G, Xiao T, Zhao M and Zhao C: CXCR4 Antagonist AMD3100 Suppresses the long-term abnormal structural changes of newborn neurons in the Intraventricular kainic acid model of epilepsy. Mol Neurobiol 53: $1518-1532,2016$

29. Yamada R and Kuba H: Structural and functional plasticity at the axon initial segment. Front Cell Neurosci 10: 250, 2016.

30. Grubb MS, Shu Y, Kuba H, Rasband MN, Wimmer VC and Bender KJ: Short- and long-term plasticity at the axon initial segment. J Neurosci 31: 16049-16055, 2011.

31. Kole MH, Letzkus JJ and Stuart GJ: Axon initial segment Kv1 channels control axonal action potential waveform and synaptic efficacy. Neuron 55: 633-647, 2007.

32. Grubb MS and Burrone J: Building and maintaining the axon initial segment. Curr Opin Neurobiol 20: 481-488, 2010.

33. Kuba H, Oichi Y and Ohmori H: Presynaptic activity regulates $\mathrm{Na}(+)$ channel distribution at the axon initial segment. Nature 465: 1075-1078, 2010

34. Wang Y, Sun D, Yue Z, Tang W, Xiao B and Feng L: Rats with malformations of cortical development exhibit decreased length of AIS and hypersensitivity to Pilocarpine-induced status epilepticus. Neurochem Res 41: 2215-2222, 2016.

35. Evans MD, Sammons RP, Lebron S, Dumitrescu AS, Watkins TB, Uebele VN, Renger JJ and Grubb MS: Calcineurin signaling mediates activity-dependent relocation of the axon initial segment. J Neurosci 33: 6950-6963, 2013.

36. Schafer DP, Jha S, Liu F, Akella T, McCullough LD and Rasband MN: Disruption of the axon initial segment cytoskeleton is a new mechanism for neuronal injury. J Neurosci 29: 13242-13254, 2009.

37. Baranauskas G, David Y and Fleidervish IA: Spatial mismatch between the $\mathrm{Na}+$ flux and spike initiation in axon initial segment. Proc Natl Acad Sci USA 110: 4051-4056, 2013.

38. Gulledge AT and Bravo JJ: Neuron morphology influences axon initial segment plasticity. eNeuro 3: pii:ENEURO.0085-15.2016, 2016.

39. Gutzmann A, Ergül N, Grossmann R, Schultz C, Wahle P and Engelhardt M: A period of structural plasticity at the axon initial segment in developing visual cortex. Front Neuroanat 8: 11, 2014.

40. Jones SL and Svitkina TM: Axon initial segment cytoskeleton: Architecture, development, and role in neuron polarity. Neural Plast 2016: 6808293, 2016. 